\title{
Nucleon resonance electrocouplings in the non-perturbative regime
}

\author{
Philip L. Cole ${ }^{* \dagger}$ \\ Idaho State University; Department of Physics; Pocatello, Idaho 83209-8106; USA \\ E-mail: colephileisu.edu
}

\section{Victor I. Mokeev}

Jefferson Lab; 12000 Jefferson Ave.; Newport News, VA 23606; USA

Skobeltsyn Nuclear Physics Institute; Moscow State University, 119899; Moscow, Russia

\section{Ralf W. Gothe}

University of South Carolina; Department of Physics; Columbia, South Carolina 29208; USA

\section{and the CLAS Collaboration}

There is an extensive search for baryon resonances using the CLAS detector in Hall B of JLab. Extracting the transition helicity amplitudes (or the $\gamma_{v} N N^{*}$ photo- and electrocouplings) sheds light on nature of the non-perturbative strong interaction. We have extended the data on differential cross sections to $Q^{2}=6.0 \mathrm{GeV}^{2}$ for the $\pi N$ electroproduction channel. Electroproduction data were also collected on the two-charged-pion channel off protons, which provides nine independent differential $\pi^{+} \pi^{-} p$ cross sections at $Q^{2}$ up to $1.5 \mathrm{GeV}^{2}$. The two-pion results, moreover, are consistent with those from independent $\pi N$ electroproduction analyses, where the background contributions in the two-pion channel are completely different from that of the single-pion one. A phenomenological approach developed at Jefferson Lab - Moscow State University is employed for separating the resonant and non-resonant contributions to the final state. The $Q^{2}$-dependent electrocouplings were then obtained for the $P_{11}(1440)$ and $D_{13}(1520)$ excited baryon states. The new data will be discussed in light of these new developments in systematically exploring the affects of meson-baryon dressing on the transition helicity amplitudes as a function of $Q^{2}$.

Sixth International Conference on Quarks and Nuclear Physics,

April 16-20, 2012

Ecole Polytechnique, Palaiseau, Paris

\footnotetext{
* Speaker.

${ }^{\dagger}$ The speaker would like to thank the organizers for setting up a wonderful conference and further thanks Bijan Saghaï and Qiang Zhao for their hard work on convening the Hadron Spectroscopy component of QNP2012. This work was supported, in part, through NSF grant PHY-0855661.
} 


\section{Introduction and Overview}

Studies of nucleon resonance electrocouplings $\gamma_{v} N N^{*}$ on exclusive meson electroproduction off nucleons represent a key part in the $\mathrm{N}^{*}$ Program with the CLAS detector $[1,2,3,4,5]$. These data enable us to explore the nature of the non-perturbative strong interaction, which is responsible for the formation of the nucleon and, in turn, the excited states of the nucleon [6]. The objectives of this $N^{*}$ research program are twofold, wherein we seek to a) extract the transition helicity amplitudes of known resonances for studying their internal structure and the interactions among constituents, which are responsible for resonance formation and b) quantify the spectroscopy of excited baryon states as well as search for new states. Both parts of this program are being pursued in various meson photo- and electroproduction channels using differential cross sections and polarization observables. Further, a means for extracting the transition helicity amplitudes in photoand electroproduction of pions has been developed by the JLab Excited Baryon Analysis Center (EBAC) within the framework of an advanced coupled-channel approach (EBAC-DCC) through a global analysis of the world's data $[9,10,11,12,13,14,15]$. In particular, from the $\pi^{0} p$ and $\pi^{+} n$ channels, EBAC-DCC has extracted the $Q^{2}$-dependent $\gamma_{v} N N^{*}$ electrocouplings for the excited states $P_{11}(1440)$ and $D_{13}(1520)$ [15]. For the two-pion mode of these excited baryons, the data [16] were analyzed within the framework of the Jefferson Lab - Moscow State University (JM) reaction model [17]. The $P_{11}(1440)$ and $D_{13}(1520)$ electrocouplings were obtained from the resonant amplitudes parametrized within the framework of a unitarized Breit-Wigner ansatz. And we remark that the two-pion analysis is consistent with results obtained in previous CLAS analyses of the $\pi^{+} n$ and $\pi^{0} p$ channels.

To satisfy the two objectives above, we first need to measure differential cross sections as well as polarization observables in both single and double pseudo-scalar production (i.e. $\pi N$, $\left.\eta p, \pi^{+} \pi^{-} p, K \Lambda, K \Sigma, \omega p, \rho^{0} p\right)$ over the full polar and azimuthal angle range. The latter two vector-meson channels, $\omega p$ are $\rho^{0} p$, are of themselves clearly not pseudo-scalars, but are identified through their pseudo-scalar decay modes. Secondly, we must determine the transition form factors (i.e. electrocouplings) of prominent excited nucleon states $\left(\mathrm{N}^{*}, \Delta^{*}\right)$ and their evolution in the range $Q^{2}<5.0 \mathrm{GeV}^{2}$; and finally, we must measure the $\mathrm{N}^{*}$ structure and its evolution with distance through the transition regime, i.e. going from the 'constituent quark region' of the combined contributions from meson-baryon dressing and the quark core at $Q^{2}<1.0 \mathrm{GeV}^{2}$ to quark-core dominance at $Q^{2}>5.0 \mathrm{GeV}^{2}$. This last condition requires the $12 \mathrm{GeV}$ energy upgrade to JLab. We next turn to the issue the transition helicity amplitudes $\left(\gamma_{v} N N^{*}\right.$ electrocouplings $)$ for the two excited baryon states $P_{11}(1440)$ and $D_{13}(1520)$ and study how they evolve with increasing $Q^{2}$.

\section{Resonance Structure: Electrocoupling and Hadronic Parameters}

We ultimately seek to establish an unambiguous relation between the information extracted phenomenologically and the non-perturbative strong interaction mechanisms that are responsible for baryon formation. From our analysis of the CLAS data on the $Q^{2}$-evolution of the $\gamma_{v} N N^{*}$ electrocouplings, we are able to describe the resonance structures in terms of an internal quark core and the surrounding meson baryon cloud, which can be viewed as originating from the reaction mechanisms as indicated in Fig. 1. Most of the single-pion (double-pion) data, upon which these 
analyses were based, were in the range $Q^{2}<5.0 \mathrm{GeV}^{2}\left(Q^{2}<0.6 \mathrm{GeV}^{2}\right)[16,18,19,20,21,22]$. As this is very short paper, we encourage the reader to review our latest $\mathrm{N}^{*}$ paper (Ref. [23] and the references therein) for further clarification on extracting the transition helicity amplitudes and attendant resonance decay parameters.
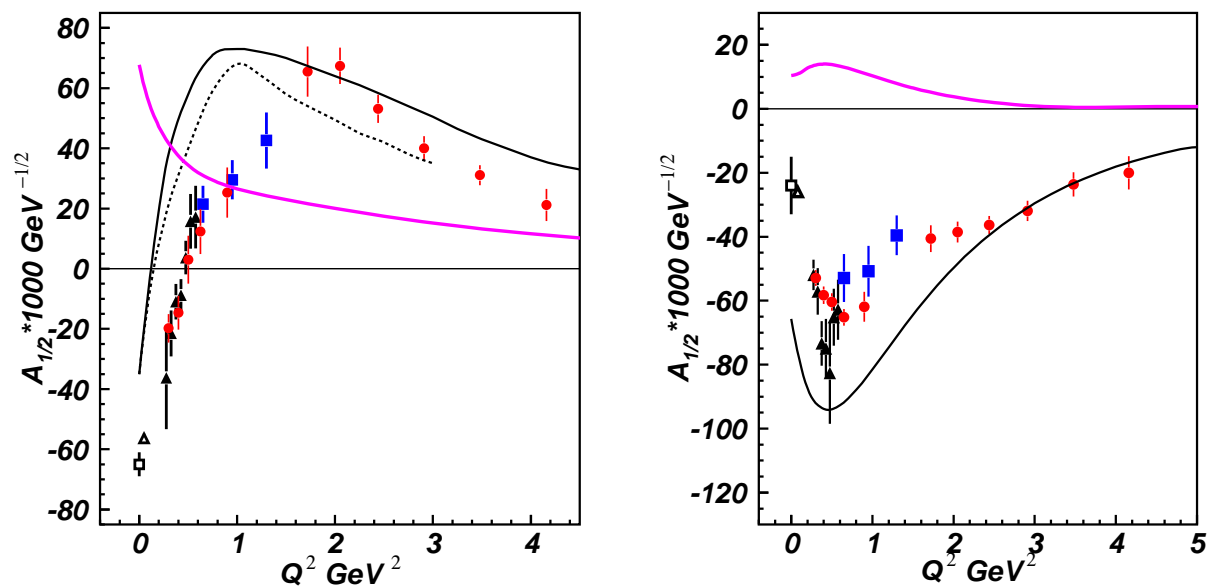

Figure 1: (color online) (Left) The $A_{1 / 2}$ electrocoupling of the $P_{11}(1440)$ state from the analyses of the $N \pi$ electroproduction data [7] (circles), $\pi^{+} \pi^{-} p$ electroproduction data [16] (triangles), and preliminary results from the $\pi^{+} \pi^{-} p$ electroproduction data at $Q^{2}$ from 0.5 to $1.5 \mathrm{GeV}^{2}$ [22] (squares). The photocouplings are taken from RPP [24] (open square) and the CLAS data analysis [25] (open triangle). Predictions from relativistic light front quark models [26, 27] are shown by black solid and dashed lines, respectively. Absolute values of meson-baryon cloud amplitudes from the EBAC-DCC coupled channel analysis [9] are shown by magenta thick solid line. (Right) The $A_{1 / 2}$ electrocoupling of the $D_{13}(1520)$ state. The data symbols are the same as in the left panel. The results of hypercentral constituent quark model [28] and absolute values of meson-baryon dressing amplitudes [9] are shown by black thin and magenta thick solid lines, respectively.

Figure 1 shows the CLAS helicity amplitude data for both single- and double-pion reactions for the two excited-baryon states. Superimposed are the contributions from the quark degrees of freedom, estimated within the framework of several quark models $[26,27,28]$ and from meson baryon dressing to the electrocouplings of the $P_{11}(1440)$ and $D_{13}(1520)$ states, estimated within the framework of the dynamical coupled channel EBAC-DCC approach [9]. These models find that the meson-baryon dressing contributions are substantial, particularly at $Q^{2}<1.0 \mathrm{GeV}^{2}$, and are quite complex, thus making it difficult to access unambiguously the quark degrees of freedom at low $Q^{2}$.

These results have further revealed that there exist two major contributions to $\gamma_{v} N N^{*}$ electrocouplings: a) an internal quark core, and b) an external meson-baryon cloud. Recent theoretical developments using the Dyson-Schwinger Equations of QCD for the interpretations of $\gamma_{v} N N^{*}$ electrocouplings provide guidelines to search for the manifestation of dynamical masses of dressed quarks. Lattice QCD is making progress toward a $\gamma_{v} N N^{*}$ electrocoupling description from the QCD Lagrangian. 


\section{Comments on the Dressed Quark Function}

It becomes apparent from Fig. 2 that for momenta larger than $2 \mathrm{GeV}$, the mass function describes a current-quark, propagating almost like a single parton. However, for momenta less than this transition boundary, the mass function rises sharply, reaching the constituent-quark mass-scale in the infrared. In this domain, the dressed-quark is far from a single parton: the effect evident in this figure is due to a cloud of low-momentum gluons attaching themselves to the current-quark. This is the phenomenon of dynamical chiral symmetry breaking. It is essentially non-perturbative and, even were the Standard Model Lagrangian to possess massless quarks, this effect would make them massive. Therefore as $Q^{2}$ tends toward zero, the effect of the meson-baryon dressing (gluon cloud) essentially endows the quark with mass. Dynamical chiral symmetry breaking is responsible for more than $97 \%$ of the nucleon's mass.

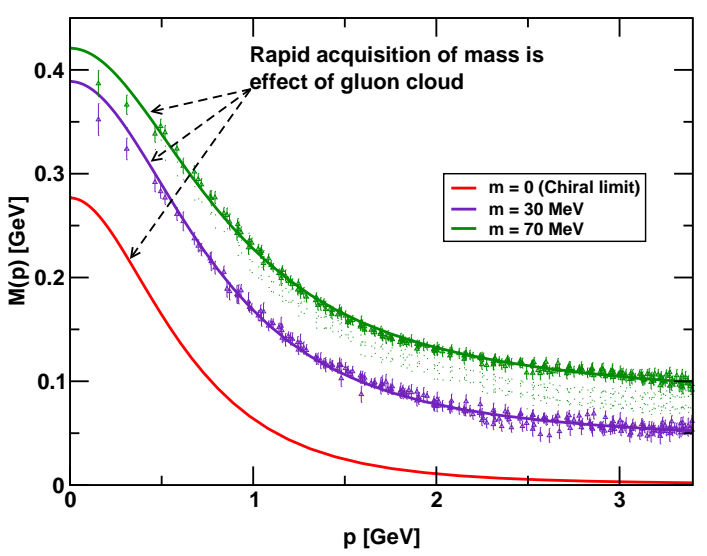

Figure 2: (color online) Dressed quark mass function, $\mathrm{M}(\mathrm{p})$, for light-quarks, obtained in Landau gauge: solid curves DSE results, including the chiral-limit [29, 30]; points with error bars are the results from unquenched LQCD [31]. The data expected from our CLAS12 $\mathrm{N}^{*}$ experiment [32] will, for the first time, allow one to study the kinematic regime for momenta running over the quark propagator for momenta $p<1.1 \mathrm{GeV}$, which spans the transition from almost-completely dressed constituent quarks to the almost-completely undressed current quarks. It is important to bear in mind that the dressed-quark propagator is gauge-covariant and hence the features evident in this figure have a genuinely measurable impact on observables.

\section{Summary}

Studying $\mathrm{N}^{*}$ s gives insight into active degrees of freedom in baryon structure at various distance scales. The 6-GeV Program offers detailed information on the transition in $\mathrm{N}^{*}$ structure from a superposition of meson-baryon and quark degrees of freedom to the quark-core dominance The quark core of the nucleon is especially important since $\mathrm{N}^{*}$ properties are determined through interactions between dressed-quarks at distances larger than those most important to the structure of ground states. Our set of experiments aims to map out the momentum-dependent dressed-quark function and thereby seek evidence for how dynamical chiral symmetry breaking in QCD generates baryonic mass. 


\section{References}

[1] I.G. Aznauryan, V.D. Burkert, T-S.H. Lee, and V.I. Mokeev, J. Phys: Conf. Ser. 299, 012008 (2011).

[2] I.G. Aznauryan and V.D. Burkert, Prog. Part. Nucl. Phys. 67, 1 (2012).

[3] V.D. Burkert, Int. J. Mod. Phys. A 26, 493 (2011).

[4] V.D. Burkert, Prog. Part. Nucl. Phys. 55, 108 (2005).

[5] V.D. Burkert and T-.S.H. Lee, Int. J. Mod. Phys. E 13, 108 (2004).

[6] I.G. Aznauryan et al., arXiv:0907.1901 [nucl-th].

[7] I.G. Aznauryan et al. (CLAS Collaboration), Phys. Rev. C 80, 055203 (2009).

[8] I.G. Aznauryan, V.D. Burkert, and V.I. Mokeev, arXiv:1108.1125.

[9] B. Julia-Diaz et al., Phys. Rev. C 77, 045205 (2008).

[10] T.-S.H. Lee, Int. J. Mod. Phys. E 18, 1215 (2009).

[11] B. Julia-Diaz et al., Phys. Rev. C 80, 025207 (2009).

[12] H. Kamano et al., Phys. Rev. C 80, 065203 (2009).

[13] H. Kamano et al., Phys. Rev. C 81, 065207 (2010).

[14] N. Suzuki et al., Phys. Rev. Lett. 104, 042302 (2010).

[15] N. Suzuki, T. Sato, and T.-S.H. Lee, Phys. Rev. C 82, 045206 (2010).

[16] G.V. Fedotov et al. (CLAS Collaboration), Phys. Rev. C 79, 015204 (2009).

[17] V.I. Mokeev et al., Phys. Rev. C 80, 045212 (2009).

[18] Theory Support for the Excited Baryon Program at the JLab 12 GeV Upgrade, JLAB-PHY-09-993, arXiv:0907.1901.

[19] The Third Workshop of the APS Topical Group in Hadron Physics April 29 - May 1, 2009, Denver, Colorado http://www.fz-juelich.de/ikp/ghp2009Program.shtml.

[20] K. Park et al. (CLAS Collaboration), Phys. Rev. C 77, 015208 (2008).

[21] I.G. Aznauryan et al., Phys. Rev. C 71, 015201 (2005).

[22] M. Ripani et al. (CLAS Collaboration), Phys. Rev. Lett. 91, 022002 (2003).

[23] V.I. Mokeev et al. (CLAS Collaboration), "A Study of the $P_{11}(1440)$ and $D_{13}(1520)$ Resonances from CLAS Data on $e p \rightarrow e^{\prime} \pi^{+} \pi^{-} p^{\prime}$," arXiv:1205.3948, submitted to Phys. Rev. C (2012).

[24] K. Nakamura et al., J. Phys. G, 37, 075021 (2010).

[25] M. Dugger et al. (CLAS Collaboration), Phys. Rev. C 79, 065206 (2009).

[26] I.G. Aznauryan, Phys. Rev. C 76, 025212 (2007).

[27] S. Capstick and B.D. Keister, Phys. Rev. D 51, 3598 (1995).

[28] M. Aiello, M M. Giannini, and E. Santopinto, J. Phys. G 24, 753 (1998).

[29] M.S. Bhagwat et al., Phys. Rev. C 68, 015203 (2003).

[30] M.S. Bhagwat and P.C. Tandy, AIP Conf. Proc. 842, 225 (2006).

[31] P.O. Bowman et al., Phys. Rev. D 71, 015203 (2005).

[32] V. Burkert, P. Cole, R. Gothe, K. Joo, V. Mokeev, and P. Stoler, cospokespersons, "Nucleon Resonance Studies with CLAS12,” Jefferson Lab CLAS12 approved experiment E12-09-003. 